\title{
Un nouvel étalon de référence pour la sensibilité spectrale de détecteur, "peu couteux" et de très faible incertitude
}

\author{
Bernard Rougié $^{1}$, Jeanne Marie Coutin ${ }^{1}$, Dominique Renoux ${ }^{2}$ \\ ${ }^{1}$ Conservatoire National des Arts et Métiers (Cnam), 61, rue du landy 93210 Saint Denis \\ ${ }^{2}$ Laboratoire National de Métrologie et d'essai (LNE), 29, rue Roger Hennequin 78190 TRAPPES
}

\begin{abstract}
New prospects in radiometric references have been introduced in 2003 by using induced junctions assembled in a particular trap arrangement and by accurately computing their internal deficiency with numerical models developed for photovoltaic cells. The claimed uncertainty, some tens of ppm, is ten times lower than the present ones. After a description of the device we present the first results confirming its abilities. We discuss the gain in uncertainty in two applications, the photometry and the pyrometry and we show the interest in this technology for disseminating radiometric references at the best level.
\end{abstract}

\section{Introduction}

Les références pour la mesure du flux de rayonnement et des grandeurs rattachées sont soit des sources calculables (de luminance ou d'intensité), soit des détecteurs (sensibilité en flux). Pour les sources, deux types de rayonnement, celui du corps noir et celui du rayonnement synchrotron sont calculables et peuvent donc servir de référence. Pour les détecteurs c'est soit les effets thermiques soit les effets quantiques du rayonnement qui sont utilisés.

Des méthodes variées font se correspondre les grandeurs caractéristiques des sources et des détecteurs.

Depuis plus de 25 ans les références métrologiques pour les mesures de flux dans les Laboratoires Nationaux de Métrologie (LNM) sont des détecteurs dits à substitution électrique fonctionnant à la température de $4 \mathrm{~K}$. Ces détecteurs, appelés 'radiomètres cryogéniques', ont une sensibilité qui s'étend de $200 \mathrm{~nm}$ dans l'ultraviolet jusqu'au domaine du teraherth, avec une incertitude maintenant bien établie de l'ordre de $10^{-4}$ dans le domaine spectral visible et proche infrarouge.

Cet étalon est la référence pour un grand nombre de grandeurs en radiométrie et photométrie, tout d'abord pour la mesure de la sensibilité des détecteurs mais aussi pour les mesures d'intensité, d'éclairement et de luminance des sources. Il a également permis la mesure par pyrométrie de températures thermodynamique au delà de $1000^{\circ} \mathrm{C}$.

Cependant, cet instrument, cher et difficile à mettre en œuvre, reste cantonné à quelques laboratoires de métrologie dans le monde. Il permet la diffusion de références de transfert de haute qualité, mais avec une inévitable dégradation des incertitudes lors du transfert.

Dans le programme de recherche européen en métrologie (EMRP) le projet 'NEWSTAR' (New Primary Standards and Traceability for Radiometry) vise à développer un étalon de haut niveau, à la fois de référence et de transfert, offrant une meilleure incertitude et une plus grande facilité d'utilisation et de dissémination jusqu'à l'utilisateur final. Cette référence est l'héritage de l'évolution des photodiodes, de la connaissance des phénomènes mis en jeu, de la technologie des semiconducteurs et des études développées pour le photovoltaïque. Les incertitudes promises théoriquement et les premiers résultats confirment l'intérêt de ces nouvelles références.

\section{Les détecteurs thermiques et quantiques}

\section{$2.1 \quad$ Les principes}

Le radiomètre cryogénique est un détecteur thermique dont l'élément sensible s'échauffe en absorbant tout ou quasiment tout le rayonnement qu'il reçoit. En l'absence de rayonnement un échauffement de ce même élément est produit en appliquant une puissance électrique de chauffage précisément mesurée. L'égalité des puissances électrique ou de rayonnement est détectée par l'égalité des échauffements.

Ce principe utilisé depuis plus d'un siècle a fait des progrès considérables dans les années 1980 du fait du fonctionnement à basse température.

La grandeur de base utilisée comme référence est le watt, unité dérivée du SI, mise en œuvre par des mesures électriques.

Une photodiode semi conductrice est un détecteur quantique, qui produit un courant photoélectrique dépendant de l'interaction de chaque photon du faisceau avec le matériau semiconducteur. Pour un détecteur idéal chaque photon interagit avec le milieu ce qui entraine le passage d'une charge électrique élémentaire par photon. Selon la loi de Planck la puissance du faisceau est proportionnelle au nombre de photons. D'autre part le courant photo-électrique généré par cette photodiode idéale est proportionnel au nombre de photons.

$$
\begin{array}{cc}
W=n \cdot h \cdot v & \mathbf{1} \\
i=n \cdot e & \mathbf{2}
\end{array}
$$




$$
\mathrm{W}=\frac{h}{e} \cdot i \cdot v \text { ou } \mathrm{W}=\frac{h}{e} \cdot i \cdot \frac{c}{\lambda}
$$

Les deux types de détecteur, thermique et photoélectrique, donnent deux méthodes de mesure de la puissance de rayonnement, le flux; L'une est rattachée au watt au travers des unités de courant et de tension électriques, la deuxième est basée sur l'unité de courant électrique et utilise le rapport e/h; constante fondamentale.

\subsection{Les incertitudes}

A la découverte des photodiodes à jonction induite[1] en 1979, les études sur des détecteurs étalon [2] ont abouti à des incertitudes de l'ordre de $1.10^{-3}$ dans un domaine restreint des longueurs d'onde visibles. Cela représentait une amélioration des incertitudes obtenues alors par des radiomètres à substitution électrique fonctionnant à température ambiante. L'arrivée du radiomètre cryogénique dans les années 1980-1990 a bouleversé cette situation en amenant des incertitudes de l'ordre de $1.10^{-4}$ dans un domaine spectral allant de l'ultraviolet à l'infrarouge lointain.

Des perspectives nouvelles ont été ouvertes en 2003 [3], en utilisant les photodiodes à jonction induite et en mettant à profit les modèles numériques de cellules photovoltaïques principalement développés pour améliorer leur rendement énergétique. Des incertitudes de l'ordre de $1.10^{-6}$ sont attendues pour ces dispositifs dans le domaine visible.

\section{L'évolution des photodiodes et leur utilisation comme référence de mesure de flux}

\subsection{Les photodiodes}

Un bref retour sur le fonctionnement des jonctions semiconductrices va expliciter l'intérêt de l'utilisation des détecteurs utilisés comme référence.

Dans un semi conducteur les électrons sont fortement liés à leur atome. La physique quantique a montré que les électrons sont pour l'essentiel d'entre eux dans une bande d'énergie appelée bande de valence qui est séparée de la bande de conduction par une bande interdite.

Un cristal de silicium est très peu conducteur car chaque atome possédant 4 électrons sur sa couche périphérique établit des liaisons de covalence avec 4 de ses voisins. Les électrons ne sont donc pas ou très peu disponibles pour la conduction électrique.

En dopant un semiconducteur pur (intrinsèque) avec des atomes donneurs d'électron (possédant 5 électrons sur leur couche périphérique) ou avec des atomes donneurs (3 électrons sur leur couche périphérique) on crée un semiconducteur de type 'n' ou 'p'. Le type 'n' possède des électrons en excès (du point de vue des liaisons de valence) et le type ' $p$ ' possède des 'absences' d'électrons, appelées trous. Ces électrons négatifs et ces trous positifs peuvent participer à la conduction électrique.
En associant ces deux types de semiconducteurs les charges mobiles, électrons de la couche ' $n$ ' ou trous de la couche ' $p$ ', se comportent comme un gaz. Elles diffusent d'une couche dans l'autre et se recombinent. Elles laissent en place les charges fixes respectivement positives et négatives. Il en résulte un champ électrique intense (quelques kilovolt/mètre) qui s'équilibre avec les charges mobiles ayant diffusé.

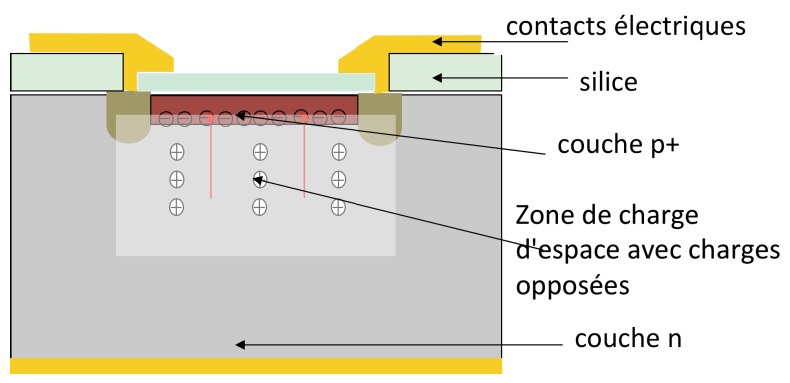

Figure 1 :Schéma de base d'une jonction semi-conductrice. Le substrat de silicium dopé ' $n$ ' $(\sim 300 \mu \mathrm{m})$ reçoit un dopage 'p' sur quelques $\mu \mathrm{m}$ puis est oxydé, ce qui crée une couche de passivation transparente.

Lorsqu'un photon assez énergétique pour exciter un atome crée une paire électron-trou dans la zone de champ électrique intense elle est rapidement dissociée par le champ électrique. L'électron et le trou sont amenés dans la zone ' $n$ ' et ' $p$ ' respectivement où ils poursuivent leur parcours sous l'effet de la diffusion ou poussés par un champ électrique créé par une polarisation électrique externe appliquée aux bornes du dispositif. C'est ce mouvement de charges qui est le courant photoélectrique.

L'efficacité d'une photodiode est conditionnée par la zone dans laquelle le rayonnement interagit. Ainsi la zone près de la surface et en arrière n'étant le siège d'aucun champ électrique interne les paires électron trou générées par le rayonnement ne se sont pas séparées et se recombinent sans générer un courant photoélectrique.

\subsection{La photodiode à couche d'inversion}

Une structure simple, la jonction induite développée en 1979 crée un champ électrique dans toute l'épaisseur du dispositif, améliorant considérablement l'efficacité de la collection des charges libres crées par le rayonnement.

Sur un silicium faiblement dopé de type 'p' un procédé particulier d'oxydation en surface piège des charges positives dans la silice $(\mathrm{SiO} 2)$ à quelques nanomètres de la surface du silicium. Les charges négatives qui apparaissent par influence électrique à la surface du silicium crée une couche de type 'n, appelée couche d'inversion. Il en résulte une jonction 'n-p' avec un champ électrique présent dès la surface. 


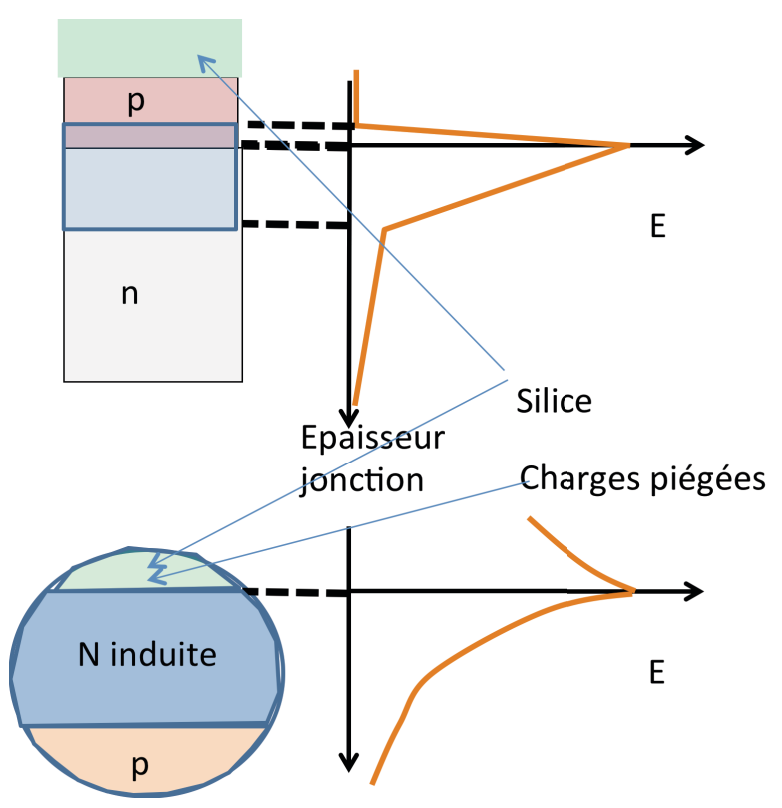

Figure 2 : Détail d'une jonction 'p-n' obtenue par diffusion de dopants et d'une jonction 'n-p' induite par piégeage de charges positives dans la couche de silice. Dans cette deuxième structure le champ électrique est maximal à la surface du silicium..

Il a été assez rapidement montré [2] que cette structure permet d'obtenir un efficacité interne voisine de l'unité, en particulier pour les longueurs d'onde les plus courtes (400 nm-650 nm) qui sont les plus rapidement absorbées.

\subsection{Principes d'une référence basée sur ces photodiodes}

Avec le développement des photodiodes de grande dimension des ensembles appelés 'pièges' (exemple en Figure 3) construits avec plusieurs .photodiodes ont montré un coefficient de réflexion global très faible (de $1.10^{-4}$ à $1.10^{-6}$ ). La lumière réfléchie n'étant pas mesurée par le détecteur, ce coefficient est nommé déficit d'efficacité externe.

Parallèlement à ces avancées, les codes de calcul pour modéliser l'efficacité des cellules photovoltaïques ont été appliqués à la structure de couche d'inversion et ont montré que le déficit d'efficacité interne calculé par ces modèles est de seulement quelques. $10^{-5}$ avec une incertitude de l'ordre de $1.10^{-6}$.

La synthèse de la situation [3] issue de la réduction du déficit de l'efficacité externe (réduction de la réflexion) et du calcul du déficit d'efficacité interne a montré une capacité à réaliser une référence quantique ayant un niveau d'incertitude de $10^{-6}$.

\section{Réalisation et validation de cette nouvelle référence}

L'instrument de référence doit satisfaire aux caractéristiques de déficits d'efficacité interne et externe présentées ci-dessus.

Le coefficient de réflexion est réduit en utilisant une structure piège constituée de deux photodiodes inclinées et ces photodiodes sont construites selon la structure de jonction induite dont les caractéristiques de dopage, de densité de charges piégées et d'épaisseur des diverses couches procurent un déficit d'efficacité interne le plus faible possible.

\subsection{Description}

La structure en piège est présentée sur la Figure 3

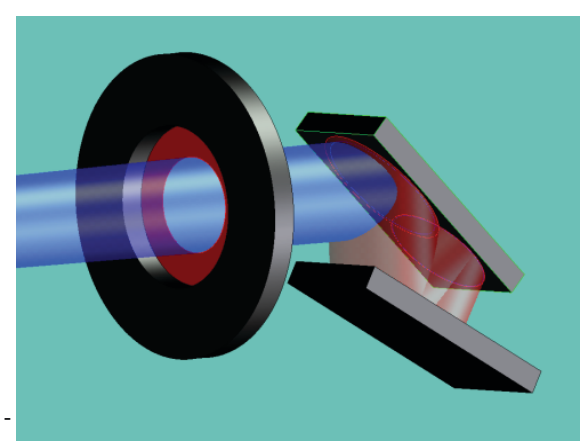

Figure 3 Vue réaliste du trajet d'un faisceau de $10 \mathrm{~mm}$ de diamètre subissant 7 réflexions dans un piège constitué de 2 photodiodes inclinées à $15^{\circ}$

Dans cette structure n'utilisant que deux photodiodes, selon l'angle que font entre elles les photodiodes, $45^{\circ}$, $22.5^{\circ}, 15^{\circ} \ldots$, le faisceau incident subit $3,5,7 \ldots$ réflexions avant son retour vers la source. La part de flux réfléchi s'amenuise à chaque réflexion. Le calcul du coefficient de réflexion global est un peu complexe puisque les angles d'incidence sont à chaque fois différents et que chaque photodiode est en fait constituée de plusieurs couches (silice dont une faible épaisseur piège des charges positives, puis silicium).

Il faut aussi noter que le coefficient de réflexion dépend de la polarisation $\mathrm{du}$ faisceau.

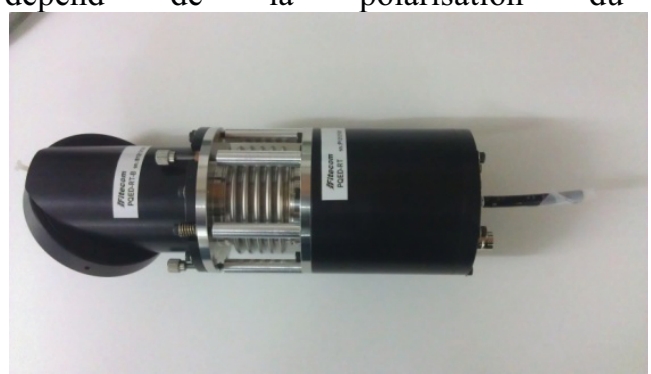

Figure 4 : $\mathrm{PQED}$ dans son enceinte pour utilisation à température ambiante

Ce détecteur peut être utilisé à température ambiante ou à $77 \mathrm{~K}$ où il donne ses meilleurs résultats. Cet instrument est généralement désigné sous le nom de PQED (Predictable Quantum Efficiency Detector) La photo d'une réalisation d'un appareil comportant ce PQED (Figure 4) montre son enceinte extérieure fermée à l'avant par une fenêtre sous incidence de Brewster, dont l'angle est ajustable pour réduire au minimum le coefficient de réflexion. La fenêtre est amovible, auquel cas un balayage de gaz propre et sec venant de l'arrière prévient toute pollution ou condensation. Le courant photoélectrique est mesuré indépendamment pour chaque photodiode. 


\subsection{Premiers résultats}

Avec un angle de $15^{\circ}$, donc 7 réflexions, le piège a un coefficient de réflexion de $45 \mathrm{ppm}$ et $17 \mathrm{ppm}$ aux longueurs d'onde de $476 \mathrm{~nm}$ et $532 \mathrm{~nm}$ avec une incertitude inférieure à 15 ppm.[4]

Le calcul du déficit d'efficacité interne calculé d'après le modèle PC1D-V5 [5] donne les valeurs de 2 ppm aux longueurs d'onde de 476 et $532 \mathrm{~nm}$, avec des incertitudes de $2 \mathrm{ppm}$. Ces valeurs sont obtenues à la température de l'azote liquide mais à la température ambiante les incertitudes restent inférieures ou de l'ordre de 100 ppm.[4]

Les mesures par ce dispositif et par un radiomètre cryogénique du flux d'un faisceau monochromatique ont été comparées dans deux expériences différentes. L'une a utilisé la méthode usuelle de mesure de la sensibilité par rapport à un radiomètre cryogénique, à savoir que le flux d'un même faisceau est mesuré successivement par le radiomètre et par le PQED, chacun comportant une fenêtre transparente pour maintenir chaque instrument sous vide et à sa température de fonctionnement. Dans l'autre expérience le PQED a été placé dans la même enceinte que le radiomètre cryogénique. De ce fait le flux à comparer n'est pas affecté par à la transmission des fenêtres du radiomètre et du PQED. Cette deuxième méthode a une incertitude de comparaison inférieure car la composante d'incertitude attachée à la connaissance de la transmission du hublot est la plus importante dans le bilan global.

Les résultats présentés sont ceux de la deuxième méthode qui a des incertitudes 3 fois inférieures à la première.

Tableau 1 Extrait de [6] (MÜLLER I. et al)

Déficit d'efficacité prédit $\Delta p(\lambda)$, et mesuré $\Delta \mathrm{m}(\lambda)$, pour 3 longueurs d'onde, à température ambiante et cryogénique et leur incertitude à $\mathbf{9 5 \%}$ de niveau de confiance.. Les bornes hautes et basses des incertitudes dissymétriques sont placées en exposant et en indice

\begin{tabular}{|c|c|c|c|c|}
\hline $\mathrm{T} / \mathrm{K}$ & $\mathrm{U}_{\text {bias }} / \mathrm{V}$ & $\lambda / \mathrm{nm}$ & $\Delta \mathrm{p}(\lambda) / \mathrm{ppm}$ & $\Delta \mathrm{m}(\lambda) / \mathrm{ppm}$ \\
\hline 299 & 5 & 476 & $39_{-9}^{+140}$ & $50 \pm 176$ \\
\hline 298 & 5 & 532 & $16_{-9}^{+130}$ & $85 \pm 60$ \\
\hline 298 & 5 & 760 & $56_{-16}^{+120}$ & $167 \pm 58$ \\
\hline 78 & 5 & 476 & $47 \square 15$ & $50 \pm 176$ \\
\hline 78 & 5 & 532 & $19 \square 10$ & $85 \pm 56$ \\
\hline 78 & 5 & 760 & $69_{-45}^{+690}$ & $656 \pm 58$ \\
\hline 78 & 16 & 760 & $43_{35}^{+120}$ & $207 \pm 58$ \\
\hline
\end{tabular}

On constate un accord global, pour un niveau de confiance de $95 \%$, entre les valeurs prédites et mesurées. L'incertitude des valeurs prédites est de $19.10^{-6}$ à la longueur d'onde de $532 \mathrm{~nm}$ à $78 \mathrm{~K}$ ce qui constitue un gain d'un facteur 5 par rapport aux mesures réalisées avec le radiomètre cryogénique.

L'autre constatation intéressante est celle de l'incertitude prédite à température ambiante $1,4.10^{-4}$ dont on constate qu'elle est à peine supérieure à celle du radiomètre cryogénique (avec son hublot d'entrée).

Le PQED à température ambiante est donc une référence qui peut être utilisée à la place du radiomètre cryogénique sans les difficultés d'utilisation de ce dernier.
Ces études sont à poursuivre pour confirmer, voire améliorer les performances et les étendre sur le domaine spectral de $400 \mathrm{~nm}$ à $900 \mathrm{~nm}$.

\section{Applications}

Parmi les applications qui découlent de l'utilisation d'un détecteur de référence de haut niveau, les mesures photométriques et les mesures de température thermodynamique par voie radiométrique connaissent des développements importants. Il est intéressant de montrer quel est l'apport de ce nouveau type de référence à ces mesures.

\subsection{Etalonnage de photomètre et radiomètre à filtre}

Les photomètres de référence, sensibles à l'éclairement lumineux, sont faits de trois éléments, un diaphragme, un filtre et un détecteur. Si le détecteur est de type piège, donc de réflexion négligeable, la sensibilité $\mathrm{du}$ photomètre est le produit de la transmission du filtre par la sensibilité du détecteur. Le filtre est conçu de façon à ce que ce produit ait la même répartition spectrale que la courbe $\mathrm{V}(\lambda)$ [7] ou en soit très proche. Dans ce produit c'est le facteur dont l'incertitude est la plus grande qui impose son incertitude. Avec les technologies actuelles c'est le filtre qui a la plus grande incertitude [8]; le fait d'utiliser un détecteur tel que le PQED, de plus faible incertitude, réduira sensiblement l'incertitude globale.

Tableau 2 Incertitudes d'un photomètre, référence primaire

\begin{tabular}{|c|c|}
\hline $\begin{array}{c}\text { Description de la composante } \\
\text { d'incertitude du photomètre }\end{array}$ & $\begin{array}{c}\mathbf{1 0}^{-4} \mathbf{x} \text { Valeur } \\
\text { relative de } \\
\text { l'incertitude type }\end{array}$ \\
\hline $\begin{array}{c}\text { Sensibilité spectrale détecteur de } \\
\text { référence }\end{array}$ & 7 \\
\hline Facteur de transmission du filtre & 11 \\
\hline Linéarité & 4 \\
\hline Surface du diaphragme & 1 \\
\hline Incertitude globale du photomètre & 15 \\
\hline
\end{tabular}

Dans une autre approche de l'étalonnage d'un photomètre, une source monochromatique éclaire alternativement le photomètre et la référence. Les facteurs d'incertitude prépondérants de ce procédé global sont la stabilité du flux, l'incertitude de la longueur d'onde et de la connaissance de la référence. Dans ce cas le PQED présente l'avantage d'une faible incertitude comparé à un détecteur piège dont l'incertitude s'accroit lors de son étalonnage par un radiomètre cryogénique. Il faut aussi noter qu'à ce niveau d'incertitude voisin de quelques $10^{-4}$, l'effet de la diffraction et de la lumière diffuse (non envisagés dans le tableau) deviennent sensibles. Le trajet du faisceau dans le PQED (taille du diaphragme, distance jusqu'au détecteur) ayant une grande similitude avec celui du trajet dans le photomètre les effets de la lumière diffuse ou de la diffraction sont partiellement compensés.

Ce procédé dont l'incertitude serait de l'ordre de quelques $10^{-4}$ est à l'étude actuellement mais il reste à valider. La stabilité à long terme de la sensibilité spectrale de l'instrument peut constituer une limitation importante. Le gain d'incertitude ainsi obtenu pourrait ne 
rester valide que quelques heures, obligeant à un processus réalisé en temps court contrairement aux méthodes actuelles se fiant à une stabilité du photomètre sur plusieurs mois voire plusieurs années.

Dans la perspective de l'évolution des sources de référence vers une technologie à LED le PEQ pourrait constituer à lui seul un photomètre si le spectre de la source, spectralement limité, est connu. Cette méthode est elle aussi à l'étude

Du fait de l'accessibilité à des incertitudes de haut niveau le PQED amène à une réduction des incertitudes mais aussi permet d'envisager des nouvelles possibilités d'étalonnage.

\subsection{Pyrométrie}

Un pyromètre est un instrument mesurant la densité spectrale de luminance. d'une surface pour en déterminer sa température

$$
L_{\lambda}(\lambda)=\frac{1}{G} \cdot \frac{d \emptyset(\square)}{\Delta \lambda}
$$

$\Phi(\lambda)$ étant le flux mesuré dans l'étendue géométrique $\mathrm{G}$ et l'intervalle spectral $\Delta \lambda$.

La température est déduite de la luminance par la loi de Planck, le rayonnement étant émis par une source à la température $\mathrm{T}$ émettant comme un corps noir, éventuellement corrigé d'un facteur d'émissivité.

Sous cette hypothèse, mesurer la température thermodynamique par voie radiométrique consiste à mesurer la luminance $L_{k}(\lambda)$ en déterminant l'étendue géométrique G, le flux $\Phi(\lambda)$ et le domaine spectral (domaine et répartition) dans lequel elle est émise.

Le tableau ci-dessous présente les incertitudes de mesure de luminance concernant la partie radiométrique.

La mesure du flux est actuellement l'une des trois composantes les plus importantes. Le PQED est l'instrument qui peut réduire l'incertitude à 1.10-4. De plus il offre un très faible taux d'inhomogénéité de la sensibilité sur sa surface.

L'étendue géométrique se calcule d'après les dimensions de la source, du récepteur et de la distance qui les sépare. Dans l'expérience relatée l"incertitude des mesures dimensionnelles est de $3.2 .10^{-4}$ mais elle est potentiellement petite car il existe quelques laboratoires proposant des incertitudes inférieures à $110^{-4}$. L'utilisation de cette capacité deviendra nécessaire en cas d'utilisation du PQED
Tableau 3 Incertitudes de mesure de luminance dans un pyromètre

\begin{tabular}{|c|c|c|}
\hline \multirow[t]{2}{*}{ Paramètres d'influence } & \multicolumn{2}{|c|}{$\begin{array}{c}10^{-4} \cdot x \text { incertitude relative } \\
\text { en }(1 \sigma)\end{array}$} \\
\hline & Actuelles & $\begin{array}{l}\text { Potentielles } \\
\text { avec PQED }\end{array}$ \\
\hline Etalon de flux & 3.3 & 1.1 \\
\hline Etendue géométrique $\mathrm{G}$ & 3.6 & 1.5 \\
\hline Longueur d'onde $\Delta \lambda$. et $\lambda$ & 0.6 & 0.6 \\
\hline $\begin{array}{c}\text { Courant photoélectrique } \\
\text { (type A) }\end{array}$ & 1.6 & 1.6 \\
\hline Etalonnages électriques & 1.4 & 1.4 \\
\hline Autres & 3.5 & 3.5 \\
\hline Global & 6.2 & 4.3 \\
\hline $\begin{array}{l}\text { Composantes dépendant de la } \\
\text { source }\end{array}$ & 1 à 5 & 1 à 5 \\
\hline
\end{tabular}

Le domaine spectral est défini par un filtre passe bande ou par un spectroradiomètre. Dans ce deuxième cas la mesure précise de la largeur spectrale (le coefficient de transmission spectrale) est difficile mais l'incertitude de $0.610^{-4}$ peut être atteinte. Pour obtenir cette performance la mesure est répétée et encadre la mesure de luminance la durée totale de luminance n'excédant pas une heure.

On ne peut pas passer sous silence les corrections de mesure de luminance provenant de la sensibilité non nulle du luminancemètre au voisinage immédiat de son champ nominal de sensibilité (responsable de ce que l'on nomme effet de taille de source). Il y a encore la sensibilité ' hors bande', le pendant spectral de l'effet de taille de source, (plus spécifiquement pour les spectroradiomètres) à savoir la sensibilité non nulle hors de la bande nominale de transmission du spectroradiomètre. L'homogénéité du détecteur et de la source monochromatique intervenant dans le processus (non décrite ici) interviennent aussi. L'ensemble de ces paramètres d'incertitude sont regroupés sous le vocable 'autre'. La valeur de leur incertitude n'est pas susceptible d'être réduite significativement et de ce fait deviendra prépondérante dans un schéma utilisant le PQED.

Les composantes dépendant de la source ne sont pas décrites ici mais peuvent être prépondérantes.

Le gain d'incertitude apporté par le PQED pour la mesure de température thermodynamique est significatif sans être révolutionnaire, mais il contribue à rendre cette mesure accessible à des laboratoires ne souhaitant pas s'équiper de l'équipement lourd nécessité par un radiomètre cryogénique

\section{Conclusion}

Le chemin suivi jusqu'à l'obtention d'une référence quantique de faible incertitude a finalement abouti près de 30 ans après les premières tentatives. Il en résulte un instrument qui a à la fois des très hautes performances à basse température mais aussi, à température ambiante, des performances comparables au radiomètre cryogénique, sans sa complexité d'utilisation.

Dans les deux exemples d'application décrits ci dessus l'introduction du PQED apporte une réduction de l'incertitude qui, sans être spectaculaire, n'en est pas moins significative. 
En raison de sa relative facilité de mise en œuvre avec des performances de haut niveau, l'emploi du PQED offre à un plus grand nombre de laboratoires de métrologie la possibilité de réaliser des références de haut niveau, en photométrie et en pyrométrie, directement reliées à la définition même des unités.

Dans la perspective quasi inéluctable de l'évolution des références photométriques vers des sources à LED le PQED est un facteur dynamisant, à la fois par ses faibles incertitudes mais aussi par le développement de nouvelles méthodes que ce document n'a pas toutes envisagées.

\section{Références}

1 HANSEN T. E., "Silicon UV-Photodiodes Using Natural Inversion Layers", Physica Scripta, Vol. 18,471475,1978. Central Institute for Industrial Research, Forskningsvn 1, Oslo 3, Norway

2 E.Zalewsk and R.Duda, Silicon photodiode device with $100 \%$ external quantum efficiencyApplied Optics Vol. 22, Issue 18, pp. 2867-2873 (1983)

3 GEIST J., BRIDA G. and RASTELLO, "Prospects for improving the accuracy of silicon photodiode selfcalibration with custom cryogenic photodiodes", Metrologia 40 (2003) S132-S135

4Meelis Sildoja et al: Predictable quantum efficient detector:I. Photodiodes and predicted responsivity, Metrologia 50 (2013) 385-394

5 CLUGSTON D.A. and BASORE P.A., 1996 PC1D version 5: 32 bit solar cell modelling on personal computers ,Proc. 26th IEEE Photovoltaic Specialists Conf. (Washington, DC) (available at http://www.pv.unsw.edu.au/pc1d)

6 MÜLLER I. et al., "Predictable quantum efficient detector : II. Characterization and confirmed responsivity", Metrologia, 50(4), 395-401, 2013

7 Le système international d'unité, 8ème édition, BIPM, p26, accessible sur

http://www.bipm.org/fr/publications/si-

brochure/download.html

8 Obein Gael, Gonzalez-Galvan Laura, Bastie Jean, Nouvelle réalisation de la candela au LNE-INM/Cnam, REVUE FRANCAISE DE METROLOGIE n¹2, Volume 2007-4 